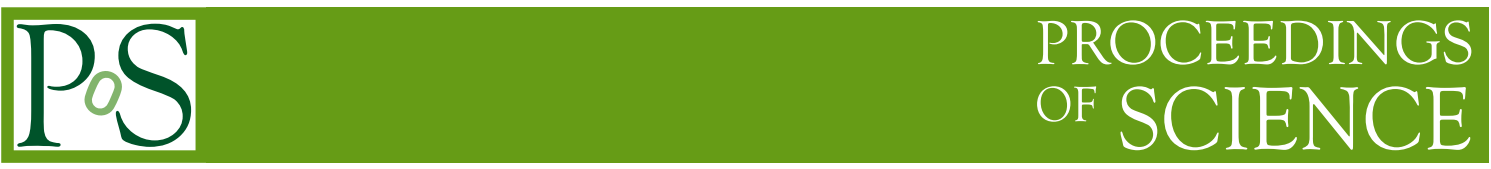

\title{
Study of a Large Forbush Decrease of July 2017
}

\section{L. Chauhanı}

Department of Physics, Govt. Model Science College

Jabalpur, M.P., INDIA

E-mail: mansukhc24@gmail.com

Vibha Chaudhary2 and M.K. Richharias

Department of Physics, Govt. Model Science College

abalpur, M.P., INDIA

E-mail: another.authorapos. it

\section{Abstract}

A large Forbush decrease (Fd) has been recorded by Moscow neutron monitoring station on 17 th July 2017. An attempt has been made to understand the probable causes which have contributed for the occurrence of the large Fd. During the period of investigation extreme solar and interplanetary events i.e. sun spot numbers, disturbance storm time (Dst) index and Ap index has been investigated. The Dst index is suddenly increased during the event period indicating high geomagnetic activity during event time. The rise in sun spot numbers earlier the onset of forbush decrease reveals that it should have some connection with the occurrence of solar flares which further effects the cosmic ray variation. Probably this decrease is a result of high activity on the sun. The forbush decrease under investigation is probably due to the solar flare generated shock wave magnetic perturbation.

Key word: Cosmic ray, forbush decrease, Dst index, Sunspot numbers. 


\section{Introduction:}

Forbush decreases (Fd's) are characterized by a rapid reduction (within a few hours) in cosmic ray (CR) intensity followed by a slow recovery typically lasting several days at $1 \mathrm{AU}$, near earth region[1]. It is a complex phenomenon, which may incorporate cosmic ray variations of interplanetary, geomagnetic and solar origin. The reduction is from about 3$20 \%$ and occurs over a timescale of several hours to a few days. A number of theoretical mechanisms have been proposed to explain the Forbush decreases dynamic sweeping of CR particles out of the inner heliosphere by the moving compression wave [2] removal of particles from the ecliptic plane by enhanced latitudinal drift [3].

The various investigations of Fd's established that these decreases are produced by perturbations in the interplanetary conditions and that these perturbations originate either from solar flares or from magnetic field structures associated with interplanetary solar wind streams. The recovery characteristics of several Fd's at 1 AU have been studied [4] and it is concluded that the recovery time is energy independent and there are no apparent changes in the recovery time with solar magnetic field reversal or with the phase of the solar cycle modulation. The recovery of the CR particles distribution seems to depend primarily upon the decay of the disturbance and only secondarily on the transport parameters of the cosmic rays.

The occurrence of Forbush decrease during the epoch of minimum solar activity period made us to look into the changes taking place over the solar disk and also in the interplanetary medium, which are the probable causes for the Forbush decrease as observed in July-2017.

\section{Observations and Analysis of Data:}

For the study of Forbush decrease event under investigation, i.e. July 2017, we have used the data from MOSCOW neutron monitoring station. The specifications of which is Latitude (deg.) 55.47, longitude (deg.) 37.32, Altitude (m) 200, and cut off rigidity (GV) 2.43. During July 2017, extreme solar and interplanetary events were observed. Data related with Ap-index, sunspot numbers and geomagnetic index Dst (daily storm time) are taken from NOAA space environment center \& world data center for geomagnetism, Kyoto University, Kyoto respectively. From all available data an effort has been made to acknowledge all possible causes that can lead to above kind of event.

\section{Result and Discussion:}

The cosmic ray intensity recorded by Moscow neutron monitoring station is shown in fig.1, for July 2017. The decrease takes place in few 
hours on $17_{\text {th }}$ July. The decrease acquires its maximum value on the same day. The total time taken from the onset of the decrease to its maximum decrease is $\sim 10$ to 15 hours. The Forbush decrease is $\sim 30 \%$ at Moscow neutron monitoring station which is at $55.47 \mathrm{deg}$. Latitude.

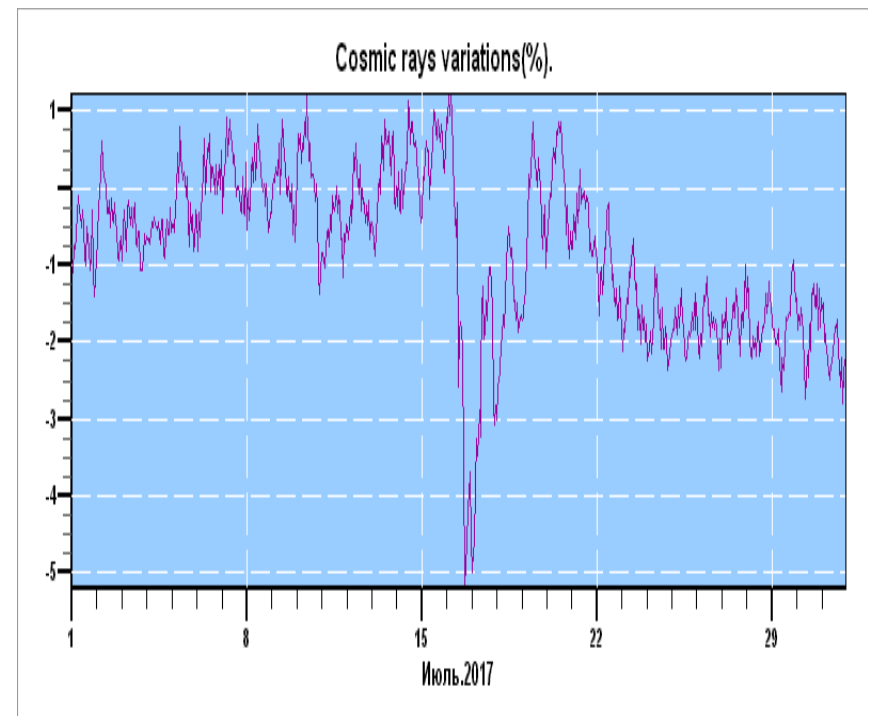

Fig. 1

The recovery phase started after July $17_{\text {th }} 2017$, which continued till the end of the month. During the recovery period large amplitude anisotropic wave trains were superimposed. The analysis of the long amplitude anisotropic wave trains during the recovery phase showed that the amplitude of this remains continuously large i.e. $4-5 \%$ after July $21_{\text {st }}$ 2017.

During the period of investigation extreme solar and interplanetary events, were observed. When the shock wave hits the earth's atmosphere, the proton flux peaked the electron flux and of course the magnetic field of earth became highly distorted. The disturbance storm time index (i.e. Dst index) is suddenly increased during the event period (fig.2), indicating high geomagnetic activity during event time. 


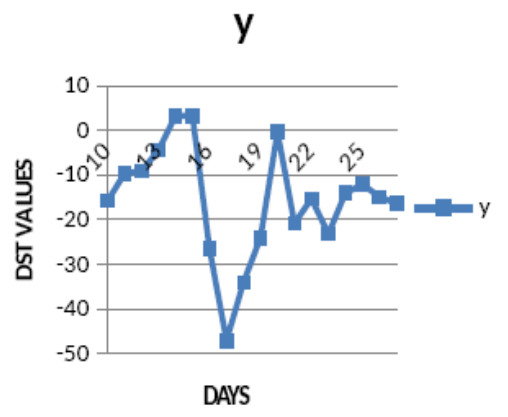

Fig.2

The variation in Ap-index and sun spot numbers for the month of July 2017 is plotted in Fig. 3 and 4 respectively. The geomagnetic activity index Ap is found to be increased during the event period. However the rise in sun spot numbers (SSN) earlier the onset of Forbush decrease reveals that it should have some connection with the occurrence of solar flares which further affects the cosmic ray variation[5]. It is clear from fig. 3 that a drastic increase in Ap-index may be one of the causes for the large Forbush decrease of July 2017.
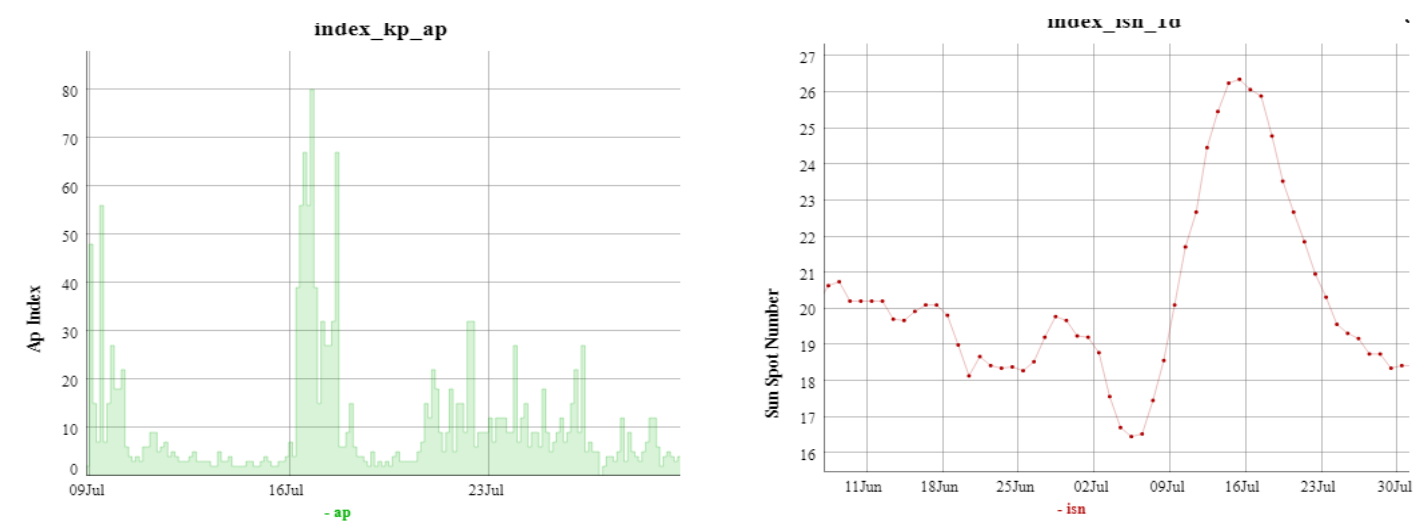

No sudden storm commencement is reported during the event time due to an intense geomagnetic activity in progress. Coronal Mass Ejections (CME's) also plays an important role in producing the Forbush decrease. However, because of non-availability of data related to CME me could not investigate its contribution. 


\section{Conclusion:}

The percentage deviation in cosmic ray intensity (called Forbush decrease) for (low latitude/high altitude) neutron monitoring station shows that this decrease is more prominent in high energy particles as compared to low energy particles. Probably this decrease is a result of high activity on the sun and in the heliosphere that look place in the during July 2017. Heightened solar activities created disturbed situations in the interplanetary space that reflected in cosmic ray behavior on the background of geomagnetic storm. The Forbush decrease under investigation is probably due to the solar flare generated shockwave magnetic perturbations. However we cannot ignore the possibility of contributions by CME's. The association of Forbush decrease with geomagnetic storm needs a detailed analysis.

Acknowledgements: The authors are indebted to various research groups working at MOSCOW neutron monitor station and the various related websites.

\section{References:}

[1] Forbush, S.E. (1938), J. Geophys. Res.43, 203.

[2] Parkar, E.N. (1963) Interplanetary dynamical process, New York.

[3] Barouch, E \& Burlaga, L.F. (1975) J. Geophys. Res.80, 449.

[4] Lockwood, J.A., Webber, W.R. \& Jokipii, J.R.(1986),

J.Geophys.Res.91, 2851.

[5] Santosh Kumar et. al., (1991) Proc.Nat.Acad.Sci., India,61(A), I. 